\title{
Some fixed-point theorems for mixed monotone operators in partially ordered probabilistic metric spaces
}

Jun $\mathrm{Wu}^{*}$

\section{"Correspondence:}

junwmath@hotmail.com

College of Mathematics and

Computer Science, Changsha

University of Science Technology,

Changsha, 410114, P.R. China

\begin{abstract}
In this paper, some fixed-point theorems for nonlinear contractive operators in partially ordered Menger probabilistic metric spaces are proved. A new extension theorem of the probabilistic versions of Boyd and Wong's nonlinear contraction theorem is presented. As a consequence, our main results improve and generalize some recent coupled fixed-point theorems and coincidence-point theorems in (Ćirić, Nonlinear Anal. 72:2009-2018, 2010; Jachymski, Nonlinear Anal., 73:2199-2203, 2010; Ćirić, Agarwal and Samet, Fixed Point Theory Appl. 2011:56, 2011).
\end{abstract}

MSC: $47 \mathrm{H} 10 ; 54 \mathrm{H} 25$

Keywords: nonlinear contractive mapping; probabilistic metric space; monotone operator; fixed point

\section{Introduction and preliminaries}

It is well known that the probabilistic version of the classical Banach contraction principle was proved in 1972 by Sehgal and Bharucha-Reid [1]. In 2010, a truthful probabilistic version of the Banach fixed-point principle for general nonlinear contractions was presented by Ljubomir Ćirić [2]. Unfortunately, there is a counterexample [3] to the Cirić's key lemma. Meanwhile, Jacek Jachymski [3] established a corrected probabilistic version of the Banach fixed-point principle for general nonlinear contractions. Also, the fixed-point theorems in probabilistic metric spaces for other contraction mappings were investigated by many authors, see [4-27] the references therein.

In this paper, we try to extend this probabilistic version theorem to the partially ordered Menger probabilistic metric spaces and establish some fixed-point theorems for monotone operators. Also, we show a sufficient and necessary condition for the uniqueness of the fixed point for a class of monotony operators. As a consequence, our main results improved and extended some recent coupled fixed-point theorems and coincidence-point theorems for mixed monotone mappings in the literature [2-4].

At this stage, we recall some well-known definitions and results in the theory of partially ordered set and probabilistic metric spaces which are used later on in the paper. For more details, we refer the reader to $[8,20]$.

Let $(X, \leq)$ be a partially ordered set, the subset $E \subset X$ is said to be a totally ordered subset if either $x \leq y$ or $y \leq x$ holds for all $x, y \in E$. We say the elements $x$ and $y$ are comparable if either $x \leq y$ or $y \leq x$ holds. It is said that the triple $(X, \leq, d)$ is a partially (totally) ordered 
complete metric space if $(X, \leq)$ is a partially (totally) ordered set and $(X, d)$ is a complete metric space. It is said that the operator $F: X \rightarrow X$ is non-decreasing monotone with respect to the order $\leq$ if for any $x, y \in X$ with $x \leq y$ we have $F(x) \leq F(y)$. Let $\Phi$ denote all the functions $\phi:[0,+\infty) \rightarrow[0,+\infty)$ which satisfy $\phi(r)<r$ and $\lim _{n \rightarrow+\infty} \phi^{n}(r)=0$ for all $r>0$.

Definition 1.1 (Bhaskar and Lakshmikantham [5]) Let $(X, \leq)$ be a partially ordered set and $F: X \times X \rightarrow X$. The mapping $F$ is said to have the mixed monotone property if $F$ is monotone non-decreasing in its first argument and is monotone non-increasing in its second argument, that is, for any $x, y \in X$,

$$
x_{1}, x_{2} \in X, \quad x_{1} \leq x_{2} \quad \Rightarrow \quad F\left(x_{1}, y\right) \leq F\left(x_{2}, y\right)
$$

and

$$
y_{1}, y_{2} \in X, \quad y_{1} \leq y_{2} \quad \Rightarrow \quad F\left(x, y_{2}\right) \leq F\left(x, y_{1}\right) .
$$

Definition 1.2 (Bhaskar and Lakshmikantham [5]) An element $(x, y) \in X^{2}$ is said to be a coupled fixed point of the mapping $F: X^{2} \rightarrow X$ if $F(x, y)=x$ and $F(y, x)=y$.

Definition 1.3 [11] Let $(X, \leq)$ be a partially ordered set and $F: X \times X \rightarrow X$ and $h: X \rightarrow X$. We say $F$ has the mixed $h$-monotone property if $F$ is monotone $h$-non-decreasing in its first argument and is monotone $h$-non-increasing in its second argument, that is, for any $x, y \in X$

$$
x_{1}, x_{2} \in X, \quad h\left(x_{1}\right) \leq h\left(x_{2}\right) \quad \text { implies } \quad F\left(x_{1}, y\right) \leq F\left(x_{2}, y\right)
$$

and

$y_{1}, y_{2} \in X, \quad h\left(y_{1}\right) \leq h\left(y_{2}\right) \quad$ implies $\quad F\left(x, y_{2}\right) \leq F\left(x, y_{1}\right)$.

Remark 1.1 If $F$ has mixed $h$-monotone property, then $F$ has mixed monotone property.

Definition 1.4 [10] A function $f:(-\infty,+\infty) \rightarrow[0,1]$ is called a distribution function if it is non-decreasing and left-continuous with $\inf _{x \in R} f(x)=0$. If in addition $f(0)=0$, then $f$ is called a distance distribution function. Furthermore, a distance distribution function $f$ satisfying $\lim _{t \rightarrow+\infty} f(t)=1$ is called a Menger distance distribution function.

The set of all Menger distance distribution functions is denoted by $\Lambda^{+}$.

Definition 1.5 [10] A triangular norm (abbreviated, $T$-norm) is a binary operation $\Delta$ on $[0,1]$, which satisfies the following conditions:

(a) $\triangle$ is associative and commutative,

(b) $\triangle$ is continuous,

(c) $\triangle(a, 1)=a$ for all $a \in[0,1]$;

(d) $\Delta(a, b) \leq \Delta(c, d)$ whenever $a \leq c$ and $b \leq d$, for each $a, b, c, d \in[0,1]$. 
Among the important examples of a $T$-norm we mention the following two $T$-norms: $\triangle_{p}(a, b)=a b$ and $\triangle_{m}(a, b)=\min \{a, b\}$. The $T$-norm $\triangle_{m}$ is the strongest $T$-norm, that is, $\triangle \leq \triangle_{m}$ for every $T$-norm $\triangle$.

Definition 1.6 [9] A triangular norm $\triangle$ is said to be of $H$-type (Hadžić type) if a family of functions $\left\{\triangle^{n}(t)\right\}_{n=1}^{+\infty}$ is equicontinuous at $t=1$, that is,

$$
\forall \varepsilon \in(0,1), \exists \delta \in(0,1): t>1-\delta \Rightarrow \triangle^{n}(t)>1-\varepsilon \quad(n \geq 1),
$$

where $\triangle^{n}:[0,1] \rightarrow[0,1]$ is defined as follows:

$$
\triangle^{1}(t)=\triangle(t, t), \quad \triangle^{n}(t)=\triangle\left(t, \triangle^{n-1}(t)\right), \quad n=2,3, \ldots
$$

Obviously, $\Delta^{n}(t) \leq t$ for any $n \in N$ and $t \in[0,1]$.

Definition 1.7 [23] A Menger probabilistic metric space (abbreviated, Menger PM space) is a triple $(X, F, \triangle)$ where $X$ is a nonempty set, $\triangle$ is a continuous $T$-norm and $F$ is a mapping from $X \times X$ into $\Lambda^{+}$such that, if $F_{p, q}$ denotes the value of $F$ at the pair $(p, q)$, the following conditions hold:

$\left(\mathrm{PM}_{1}\right) F_{p, q}(t)=1$ for all $t>0$ if and only if $p=q(p, q \in X)$.

$\left(\mathrm{PM}_{2}\right) F_{p, q}(t)=F_{q, p}(t)$ for all $p, q \in X$ and $t>0$.

$\left(\mathrm{PM}_{3}\right) F_{p, r}(s+t) \geq \triangle\left(F_{p, q}(t), F_{q, r}(s)\right)$ for all $p, q, r \in X$ and every $s>0, t>0$.

Definition 1.8 [23] A sequence $\left\{x_{n}\right\}$ in $X$ is said to converge to a point $x$ in $X$ (written as $\left.x_{n} \rightarrow x\right)$ if for every $\delta>0$ and $\lambda \in(0,1)$, there is an integer $N(\delta, \lambda)>0$ such that $F_{x_{n}, x}(\delta)>$ $1-\lambda$, for all $n \geq N(\delta, \lambda)$. The sequence is said to be a Cauchy sequence if for each $\delta>$ 0 and $\lambda \in(0,1)$, there is an integer $N(\delta, \lambda)>0$ such that $F_{x_{n}, x_{m}}(\delta)>1-\lambda$, for all $n, m \geq$ $N(\delta, \lambda)$. A Menger PM space $(X, F, \triangle)$ is said to be complete if every Cauchy sequence in $X$ converges to a point of $X$.

\section{Main results}

Lemma 2.1 Let $(X, F, \triangle)$ be a Menger $P M$ space and $\varphi \in \Phi$. If

$$
F_{p, q}(\varphi(t))=F_{p, q}(t), \text { for all } t>0,
$$

then $p=q$.

Proof Since $F_{p, q}(\varphi(t))=F_{p, q}(t)$, then $F_{p, q}\left(\varphi^{n}(t)\right)=F_{p, q}(t)$ for all $n \in Z^{+}$and $t>0$. It follows from $\lim _{t \rightarrow+\infty} F_{p, q}(t)=1$ that, for $\varepsilon \in(0,1)$, there is $t_{0}>0$ such that $F_{p, q}\left(t_{0}\right)>1-\varepsilon$. Also, for $\delta>0$, there is $N(\delta) \in Z^{+}$such that $\varphi^{n}\left(t_{0}\right) \leq \delta$ for all $n \geq N(\delta)$. Thus

$$
F_{p, q}(\delta) \geq F_{p, q}\left(\varphi^{n}\left(t_{0}\right)\right) \geq F_{p, q}\left(t_{0}\right)>1-\varepsilon .
$$

This implies that $F_{p, q}(t)=1$ for all $t>0$. Thus $p=q$. 
Lemma 2.2 Let $n \geq 1$. If $F \in \Lambda^{+}, g_{1}, g_{2}, \ldots, g_{n}: R \rightarrow[0,1]$ and, for some $\varphi \in \Phi$,

$$
F(\varphi(t)) \geq \min \left\{g_{1}(t), g_{2}(t), \ldots, g_{n}(t), F(t)\right\}, \quad \text { for all } t>0,
$$

then $F(\varphi(t)) \geq \min \left\{g_{1}(t), g_{2}(t), \ldots, g_{n}(t)\right\}$ for all $t>0$.

Proof If $\min \left\{g_{1}(t), g_{2}(t), \ldots, g_{n}(t), F(t)\right\}<F(t)$, then the result of Lemma 2.2 trivially holds.

If $\min \left\{g_{1}(t), g_{2}(t), \ldots, g_{n}(t), F(t)\right\}=F(t)$, then $F(t) \leq F(\varphi(t)) \leq F(t)$. Thus $F(\varphi(t))=F(t)$ for all $t>0$. By the proof of Lemma 2.1, we see that $F(t)=1$ for all $t>0$. Thus $g_{1}(t)=g_{2}(t)=$ $\cdots=g_{n}(t)=1$ for all $t>0$. Then $F(\varphi(t)) \geq \min \left\{g_{1}(t), g_{2}(t), \ldots, g_{n}(t)\right\}$ for all $t>0$. The proof is complete.

For $\tilde{a}=(x, y), \tilde{b}=(u, v) \in X^{2}$, we introduce a distribution function $\tilde{F}$ from $X^{2}$ into $\Lambda^{+}$ defined by

$$
\tilde{F}_{\tilde{a}, \tilde{b}}(t)=\min \left\{F_{x, u}(t), F_{y, v}(t)\right\} \quad \text { for all } t>0
$$

Lemma 2.3 If $(X, F, \triangle)$ is a complete Menger PM space, then $\left(X^{2}, \tilde{F}, \triangle\right)$ is also a complete Menger PM space.

Proof It is sufficient to prove that, for $\tilde{a}=(x, y), \tilde{b}=(u, v), \tilde{c}=(p, q) \in X^{2}$ and $t, s \geq 0$,

$$
\tilde{F}_{\tilde{a}, \tilde{b}}(t+s) \geq \triangle\left(\tilde{F}_{\tilde{a}, \tilde{c}}(t), \tilde{F}_{\tilde{c}, \tilde{b}}(s)\right)
$$

In fact, by direct computation, we have

$$
\begin{aligned}
\tilde{F}_{\tilde{a}, \tilde{b}}(t+s) & =\min \left\{F_{x, u}(t+s), F_{y, v}(t+s)\right\} \\
& \geq \min \left\{\triangle\left(F_{x, p}(t), F_{p, u}(s)\right), \triangle\left(F_{y, q}(t), F_{q, v}(s)\right)\right\} \\
& \geq \triangle\left(\min \left\{F_{x, p}(t), F_{y, q}(t)\right\}, \min \left\{F_{p, u}(s), F_{q, v}(s)\right\}\right) \\
& =\triangle\left(\tilde{F}_{\tilde{a}, \tilde{c}}(t), \tilde{F}_{\tilde{c}, \tilde{b}}(s)\right) .
\end{aligned}
$$

If the sequence $\left\{\tilde{x}_{n}\right\}=\left\{\left(x_{n}, y_{n}\right)\right\}$ is a Cauchy sequence in $\left(X^{2}, \tilde{F}, \Delta\right)$, then, for all $t>0$, every $\delta>0$ and $\lambda \in(0,1)$, there is a positive integer $N(\delta, \lambda)>0$ such that

$$
\tilde{F}_{\tilde{x}_{n}, \tilde{x}_{m}}(t)=\min \left\{F_{x_{n}, x_{m}}(t), F_{y_{n}, y_{m}}(t)\right\}>1-\lambda, \quad \text { for all } n, m>N(\delta, \lambda) \text {. }
$$

Then $F_{x_{n}, x_{m}}(t)>1-\lambda$ and $F_{y_{n}, y_{m}}(t)>1-\lambda$. Thus both $\left\{x_{n}\right\}$ and $\left\{y_{n}\right\}$ are Cauchy sequences in $(X, F, \triangle)$. Following Definition 1.8, it is a standard argument to show that the Cauchy sequence $\left\{\tilde{x}_{n}\right\}$ converges to a point of $X^{2}$. Thus $\left(X^{2}, \tilde{F}, \triangle\right)$ is a complete Menger PM space. The proof is complete.

Theorem 2.1 Let $(X, \preceq, F, \triangle)$ be a partially ordered complete Menger PM space with a $T$-norm $\triangle$ of H-type. Suppose $G: X \rightarrow X$ is a non-decreasing operator with respect to the order $\preceq$ on X. Assume 
(i) there is a $\varphi \in \Phi$ such that, for all $t>0$ and $x, y \in X$ with $x \preceq y$,

$$
F_{G(x), G(y)}(\varphi(t)) \geq \min \left\{F_{x, y}(t), F_{x, G(x)}(t), F_{y, G(y)}(t)\right\}
$$

(ii) there exists an $x_{0} \in X$ such that $x_{0} \preceq G\left(x_{0}\right)$;

(iii) either if (a) $G$ is a continuous operator or (b) if a non-decreasing monotone sequence $x_{n}$ in $X$ tends to $\bar{x}$, then $x_{n} \preceq \bar{x}$ for all $n$.

Then the operator $G$ has a fixed point in $X$.

Proof Define a sequence $\left\{x_{n}\right\} \subset X$ by $x_{n+1}=G\left(x_{n}\right), n=0,1, \ldots$ Noting $x_{0} \preceq G\left(x_{0}\right)$ and the monotony of $G$, we have

$$
x_{0} \preceq x_{1} \preceq x_{2} \preceq \cdots \preceq x_{n} \preceq \cdots
$$

If there exists $n_{0}$ such that $x_{n_{0}}=x_{n_{0}+1}$, then $x_{n_{0}}=G\left(x_{n_{0}}\right)$ and $x_{n_{0}}$ is a fixed point of $G$. Then the result of Theorem 2.1 trivially holds.

Suppose now that $x_{n} \neq x_{n+1}$ for all $n$. Following the assumption (i), we see that

$$
\begin{aligned}
F_{x_{n+1}, x_{n+2}}(\varphi(t)) & =F_{G\left(x_{n}\right), G\left(x_{n+1}\right)}(\varphi(t)) \\
& \geq \min \left\{F_{x_{n}, x_{n+1}}(t), F_{x_{n}, G\left(x_{n}\right)}(t), F_{x_{n+1}, G\left(x_{n+1}\right)}(t)\right\} \\
& =\min \left\{F_{x_{n}, x_{n+1}}(t), F_{x_{n}, x_{n+1}}(t), F_{x_{n+1}, x_{n+2}}(t)\right\} \\
& =\min \left\{F_{x_{n}, x_{n+1}}(t), F_{x_{n+1}, x_{n+2}}(t)\right\} .
\end{aligned}
$$

It follows from $\varphi \in \Phi$ and Lemma 2.2 that

$$
F_{x_{n+1}, x_{n+2}}(\varphi(t)) \geq F_{x_{n}, x_{n+1}}(t) \text { for all } t>0 \text {. }
$$

Thus we have

$$
F_{x_{n+1}, x_{n+2}}\left(\varphi^{n+1}(t)\right) \geq F_{x_{0}, x_{1}}(t) \text { for all } t>0 \text {. }
$$

For $\delta>0$ and $\varepsilon \in(0,1)$, since $\lim _{t \rightarrow+\infty} F_{x_{0}, x_{1}}(t)=1$, there is a $t_{0}$ such that $F_{x_{0}, x_{1}}\left(t_{0}\right)>1-\varepsilon$. Also, by $\lim _{n \rightarrow \infty} \varphi^{n}\left(t_{0}\right)=0$, there is a $N_{0}$ such that $\varphi^{n}\left(t_{0}\right)<\delta$ for $n \geq N_{0}$. Thus we obtain

$$
F_{x_{n+1}, x_{n+2}}(\delta) \geq F_{x_{n+1}, x_{n+2}}\left(\varphi^{n+1}\left(t_{0}\right)\right) \geq F_{x_{0}, x_{1}}\left(t_{0}\right)>1-\varepsilon \quad \text { for } n>N_{0} .
$$

This means $\lim _{n \rightarrow \infty} F_{x_{n+1}, x_{n+2}}(t)=1$ for all $t>0$.

Next we should prove that the sequence $\left\{x_{n}\right\}$ is a Cauchy sequence in $X$. It is necessary to prove that, for any $\delta>0$ and $\varepsilon \in(0,1)$, there is $N(\varepsilon, \delta)$ such that

$$
F_{x_{n}, x_{m}}(\delta)>1-\varepsilon \quad \text { for all } m>n \geq N(\varepsilon, \delta) \text {. }
$$

To this end, firstly, we can show the following inequality by mathematical induction:

$$
F_{x_{n+k}, x_{n}}(\delta) \geq \Delta^{k}\left(F_{x_{n}, x_{n+1}}(\delta-\varphi(\delta))\right) \text { for all } k \geq 1
$$


As $k=1$,

$$
\begin{aligned}
F_{x_{n+1}, x_{n}}(\delta) & \geq F_{x_{n+1}, x_{n}}(\delta-\varphi(\delta)) \\
& =\triangle\left(F_{x_{n}, x_{n+1}}(\delta-\varphi(\delta)), 1\right) \\
& \geq \triangle\left(F_{x_{n}, x_{n+1}}(\delta-\varphi(\delta)), F_{x_{n}, x_{n+1}}(\delta-\varphi(\delta))\right) \\
& =\triangle^{1}\left(F_{x_{n}, x_{n+1}}(\delta-\varphi(\delta))\right) .
\end{aligned}
$$

Thus (2) holds for $k=1$.

Now we assume (2) holds for $1 \leq k \leq p$. When $k=p+1$,

$$
F_{x_{n+p+1}, x_{n}}(\delta) \geq \triangle\left(F_{x_{n+1}, x_{n}}(\delta-\varphi(\delta)), F_{x_{n+1}, x_{n+p+1}}(\varphi(\delta))\right) .
$$

Following the formulation (1), it is a standard argument (by contradiction) to show that $F_{x_{n+1}, x_{n+2}}(\delta) \geq F_{x_{n}, x_{n+1}}(\delta)$ for all $n$. Thus $F_{x_{n+p+1}, x_{n+p}}(\delta) \geq F_{x_{n}, x_{n+1}}(\delta)$. Then we have

$$
\begin{aligned}
F_{x_{n+1}, x_{n+p+1}}(\varphi(\delta)) & =F_{G\left(x_{n}\right), G\left(x_{n+p}\right)}(\varphi(\delta)) \\
& \geq \min \left\{F_{x_{n}, x_{n+p}}(\delta), F_{x_{n}, x_{n+1}}(\delta), F_{x_{n+p}, x_{n+p+1}}(\delta)\right\} \\
& =\min \left\{F_{x_{n}, x_{n+p}}(\delta), F_{x_{n}, x_{n+1}}(\delta)\right\} \\
& \geq \min \left\{\triangle^{p}\left(F_{x_{n}, x_{n+1}}(\delta-\varphi(\delta))\right), F_{x_{n}, x_{n+1}}(\delta-\varphi(\delta))\right\} \\
& =\triangle^{p}\left(F_{x_{n}, x_{n+1}}(\delta-\varphi(\delta))\right) .
\end{aligned}
$$

Then

$$
\begin{aligned}
F_{x_{n+p+1}, x_{n}}(\delta) & \geq \triangle\left(F_{x_{n+1}, x_{n}}(\delta-\varphi(\delta)), \triangle^{p}\left(F_{x_{n}, x_{n+1}}(\delta-\varphi(\delta))\right)\right) \\
& =\triangle^{p+1}\left(F_{x_{n}, x_{n+1}}(\delta-\varphi(\delta))\right) .
\end{aligned}
$$

Thus

$$
F_{x_{n+k}, x_{n}}(\delta) \geq \Delta^{k}\left(F_{x_{n}, x_{n+1}}(\delta-\varphi(\delta))\right) \quad \text { for all } k \geq 1
$$

Noting the $T$-norm $\triangle$ of $H$-type, for a given $\varepsilon \in(0,1)$, there exists $\lambda \in(0,1)$ such that $\triangle^{n}(t)>1-\varepsilon$ for all $n \geq 1$ and when $t>1-\lambda$. On the other hand, by $\lim _{n \rightarrow+\infty} F_{x_{n}, x_{n+1}}(\delta-$ $\varphi(\delta))=1$, there is a $N_{1}(\varepsilon, \delta)$ such that

$$
F_{x_{n}, x_{n+1}}(\delta-\varphi(\delta))>1-\lambda \quad \text { for all } n>N_{1}(\varepsilon, \delta) .
$$

Thus

$$
F_{x_{n+k}, x_{n}}(\delta) \geq 1-\varepsilon \quad \text { for all } k \geq 1 \text { and } n>N_{1}(\varepsilon, \delta) .
$$

This implies that the sequence $\left\{x_{n}\right\}$ is a Cauchy sequence in $X$. Then by the completeness of $X$, there is $\bar{x} \in X$ such that $\lim _{n \rightarrow+\infty} x_{n}=\bar{x}$. 
Suppose (a) holds. It follows from $x_{n+1}=G\left(x_{n}\right)$ that

$$
\bar{x}=\lim _{n \rightarrow+\infty} x_{n+1}=\lim _{n \rightarrow+\infty} G\left(x_{n}\right)=G(\bar{x}) .
$$

Then the operator $G$ has a fixed point in $X$.

Suppose (b) in the assumption (iii) holds, then $x_{n} \preceq \bar{x}$ and we have

$$
F_{x_{n+1}, G(\bar{x})}(\varphi(t))=F_{G\left(x_{n}\right), G(\bar{x})}(\varphi(t)) \geq \min \left\{F_{x_{n}, \bar{x}}(t), F_{x_{n}, x_{n+1}}(t), F_{\bar{x}, G(\bar{x})}(t)\right\} .
$$

Letting $n$ go to infinity, we have $F_{\bar{x}, G(\bar{x})}(\varphi(t)) \geq F_{\bar{x}, G(\bar{x})}(t)$. It follows from Lemma 2.1 that $\bar{x}=$ $G(\bar{x})$. Then the operator $G$ has a fixed point in $X$. The proof of Theorem 2.1 is complete.

Let $D=\{x \in X: x$ and $G(x)$ are comparable $\}$, then we have the following.

Theorem 2.2 Let $(X, \preceq, F, \triangle)$ be a partially ordered complete Menger PM space with a $T$-norm $\triangle$ of H-type. Suppose $G: X \rightarrow X$ is a non-decreasing operator with respect to the order $\preceq$ on X. Assume (i) in Theorem 2.1 and one of following conditions hold:

(a) $G$ is a continuous operator;

(b) if a monotone sequence $x_{n}$ in $X$ tends to $\bar{x}$, then $x_{n}$ and $\bar{x}$ are comparable for all $n$.

Then the operator $G$ has a fixed point in $X$ if and only if $D \neq \phi$. Furthermore, if $D$ is a totally ordered nonempty subset, then the operator $G$ has a unique fixed point in $X$

Proof $\Rightarrow$ : It is easy to see that all the fixed points of $G$ fall in the set $D$. Thus if the operator $G$ has a fixed point in $X$, then $D \neq \phi$.

$\Leftarrow$ : If $D \neq \phi$ and $x_{0} \in D$, then there are two cases: $x_{0} \preceq G\left(x_{0}\right)$ and $G\left(x_{0}\right) \preceq x_{0}$. For the former case, following Theorem 2.1, we claim that the operator $G$ has a fixed point in $X$. For the latter case: $G\left(x_{0}\right) \preceq x_{0}$, noting the symmetry of the probabilistic metric, we see that (1) holds for $y \preceq x$. Thus

$$
F_{G(x), G(y)}(\varphi(t)) \geq \min \left\{F_{x, y}(t), F_{x, G(x)}(t), F_{y, G(y)}(t)\right\}
$$

for each $x, y \in X$ satisfying $x$ is comparable with $y$. Constructing the sequence $\left\{x_{n}\right\}$ in $X$ by $x_{n}=G\left(x_{n-1}\right)$, for $n=1,2, \ldots$, we have

$$
\cdots \preceq x_{n} \preceq \cdots \preceq x_{2} \preceq x_{1} \preceq x_{0} .
$$

Following a similar proof as of Theorem 2.1, we conclude that the operator $G$ has a fixed point $\bar{x}$ in $X$.

Finally, we suppose that $D$ is a totally ordered nonempty subset. It is sufficient to prove the uniqueness of fixed point of $\tilde{F}$. Let $x$ and $y$ be two fixed points of $G$, then $x$ is comparable with $y, G(x)=x$ and $G(y)=y$. Following the assumption (i), we have

$$
\begin{aligned}
F_{x, y}(\varphi(t)) & =F_{G(x), G(y)}(\varphi(t)) \\
& \geq \min \left\{F_{x, y}(t), F_{x, G(x)}(t), F_{y, G(y)}(t)\right\} \\
& =F_{x, y}(t) .
\end{aligned}
$$


On the other hand, by $\varphi(t)<t$ and the monotony of $F_{x, y}$, we see that $F_{x, y}(t) \geq F_{x, y}(\varphi(t))$. Thus $F_{x, y}(t)=F_{x, y}(\varphi(t))$. It follows from Lemma 2.1 that $x=y$. The proof of Theorem 2.2 is complete.

Corollary 2.1 Let $(X, \leq, F, \triangle)$ be a partially ordered complete Menger PM space with a $T$-norm $\triangle$ of H-type. Suppose $A: X \times X \rightarrow X$ is a mapping satisfying the mixed monotone property on $X$ and, for some $\varphi \in \Phi$,

$$
\begin{aligned}
F_{A(x, y), A(u, v)}(\varphi(t)) \geq & \min \left\{F_{x, u}(t), F_{y, v}(t), F_{x, A(x, y)}(t),\right. \\
& \left.F_{u, A(u, v)}(t), F_{y, A(y, x)}(t), F_{v, A(v, u)}(t)\right\}
\end{aligned}
$$

for all $x, y \in X$ for which $x \leq u$ and $v \leq y$ and all $t>0$. Suppose that $A$ is a continuous mapping or $X$ has the following properties:

(i) if non-decreasing sequence $x_{n}$ tends to $x$, then $x_{n} \leq x$ for all $n$,

(ii) if non-increasing sequence $y_{n}$ tends to $y$, then $y \leq y_{n}$ for all $n$.

If there exist $x_{0}, y_{0} \in X$ such that $x_{0} \leq A\left(x_{0}, y_{0}\right)$ and $A\left(y_{0}, x_{0}\right) \leq y_{0}$, then $A$ has a coupled point, that is, there exist $p, q \in X$ such that $A(p, q)=p$ and $A(q, p)=q$.

Proof Let $\tilde{X}=X \times X$, for $\tilde{a}=(x, y), \tilde{b}=(u, v) \in x$, we introduce the order $\preceq$ as

$$
\tilde{a} \preceq \tilde{b} \quad \Leftrightarrow \quad x \leq u, \quad v \leq y .
$$

It follows from Lemma 2.3 that $(\tilde{X}, \preceq, \tilde{F}, \triangle)$ is also a partially ordered complete Menger PM space, where

$$
\tilde{F}_{\tilde{a}, \tilde{b}}(t):=\min \left\{F_{x, u}(t), F_{y, v}(t)\right\} .
$$

The self-mapping $G: \tilde{X} \rightarrow \tilde{X}$ is given by

$$
G(\tilde{a})=(A(x, y), A(y, x)) \quad \text { for all } \tilde{a}=(x, y) \in \tilde{X} \text {. }
$$

Then a coupled point of $A$ is a fixed point of $G$ and vice versa.

If $\tilde{a} \preceq \tilde{b}$, then $x \leq u$ and $v \leq y$. Noting the mixed monotone property of $A$, we see that $A(x, y) \leq A(u, v)$ and $A(v, u) \leq A(y, x)$, then $G(\tilde{a}) \preceq G(\tilde{b})$. Thus $G$ is a non-decreasing operator with respect to the order $\preceq$ on $\tilde{X}$.

On the other hand, for all $t>0$ and $\tilde{a}=(x, y), \tilde{b}=(u, v) \in \tilde{X}$ with $\tilde{a} \preceq \tilde{b}$, we have

$$
\begin{aligned}
F_{A(x, y), A(u, v)}(\varphi(t)) \geq & \min \left\{F_{x, u}(t), F_{y, v}(t), F_{x, A(x, y)}(t),\right. \\
& \left.F_{u, A(u, v)}(t), F_{y, A(y, x)}(t), F_{v, A(v, u)}(t)\right\} \\
= & \min \left\{\min \left\{F_{x, u}(t), F_{y, v}(t)\right\}, \min \left\{F_{x, A(x, y)}(t), F_{u, A(u, v)}(t)\right\},\right. \\
& \left.\min \left\{F_{y, A(y, x)}(t), F_{v, A(v, u)}(t)\right\}\right\} \\
= & \min \left\{\tilde{F}_{\tilde{a} \tilde{b}}(t), \tilde{F}_{\tilde{a}, G(\tilde{a})}(t), \tilde{F}_{\tilde{b}, G(\tilde{b})}(t)\right\} .
\end{aligned}
$$

Similarly,

$$
F_{A(y, x), A(v, u)}(\varphi(t)) \geq \min \left\{\tilde{F}_{\tilde{a}, \tilde{b}}(t), \tilde{F}_{\tilde{a}, G(\tilde{a})}(t), \tilde{F}_{\tilde{b}, G(\tilde{b})}(t)\right\} .
$$


Thus

$$
\tilde{F}_{G(\tilde{a}), G(\tilde{b})}(\varphi(t)) \geq \min \left\{\tilde{F}_{\tilde{a}, \tilde{b}}(t), \tilde{F}_{\tilde{a}, G(\tilde{a})}(t), \tilde{F}_{\tilde{b}, G(\tilde{b})}(t)\right\}
$$

Also, there exists an $\tilde{x}_{0}=\left(x_{0}, y_{0}\right) \in \tilde{X}$ such that $\tilde{x}_{0} \preceq G\left(\tilde{x}_{0}\right)=\left(A\left(x_{0}, y_{0}\right), A\left(y_{0}, x_{0}\right)\right)$.

If a non-decreasing monotone sequence $\left\{\tilde{x}_{n}\right\}=\left\{\left(x_{n}, y_{n}\right)\right\}$ in $\tilde{X}$ tends to $\tilde{x}=(x, y)$, then $\tilde{x}_{n}=$ $\left(x_{n}, y_{n}\right) \preceq\left(x_{n+1}, y_{n+1}\right)=\tilde{x}_{n+1}$, that is, $x_{n} \leq x_{n+1}$ and $y_{n+1} \leq y_{n}$. Thus $\left\{x_{n}\right\}$ is non-decreasing sequence tending to $x$ and $\left\{y_{n}\right\}$ a non-increasing sequence tending to $y$. Thus $x_{n} \leq x$ and $y \leq y_{n}$ for all $n$. This implies $\tilde{x}_{n} \preceq \tilde{x}$. Obviously, the continuity of $A$ implies the continuity of $G$.

Following Theorem 2.1, we see that $A$ has a coupled point, that is, there exist $p, q \in X$ such that $A(p, q)=p$ and $A(q, p)=q$.

Corollary 2.2 Let $(X, \preceq, F, \triangle)$ be a partially ordered complete Menger PM space with a $T$-norm $\triangle$ of H-type. Suppose $A: X \times X \rightarrow X$ and $h: X \rightarrow X$ are two mappings such that $A$ has the h-mixed monotone property on $X$ and, for some $\varphi \in \Phi$,

$$
\begin{aligned}
F_{A(x, y), A(u, v)}(\varphi(t)) \geq & \min \left\{F_{h(x), h(u)}(t), F_{h(y), h(v)}(t), F_{h(x), A(x, y)}(t),\right. \\
& \left.F_{h(u), A(u, v)}(t), F_{h(y), A(y, x)}(t), F_{h(v), A(v, u)}(t)\right\}
\end{aligned}
$$

for all $x, y \in X$ for which $h(x) \leq h(u)$ and $h(v) \leq h(y)$ and all $t>0$. Suppose also that $A(X \times$ $X) \subset h(X), h(X)$ is closed and

if $\left\{h\left(x_{n}\right)\right\} \subset X$ is a non-decreasing sequence with $h\left(x_{n}\right) \rightarrow h(z)$ in $h(X)$

then $h\left(x_{n}\right) \leq h(z)$ for all $n$ holds,

if $\left\{h\left(y_{n}\right)\right\} \subset X$ is a non-increasing sequence with $h\left(y_{n}\right) \rightarrow h(z)$ in $h(X)$

then $h(z) \leq h\left(y_{n}\right)$ for all $n$ holds.

If there exist $x_{0}, y_{0} \in X$ such that $h\left(x_{0}\right) \leq A\left(x_{0}, y_{0}\right)$ and $A\left(y_{0}, x_{0}\right) \leq h\left(y_{0}\right)$, then $A$ and $h$ have a coupled coincidence point, that is, there exist $p, q \in X$ such that $A(p, q)=h(p)$ and $A(q, p)=h(q)$.

Proof Using the method in [7], we see that there exists $E \subset X$ such that $h(E)=h(X)$ and $h: E \rightarrow X$ is one-to-one. Define the mapping $H: h(E) \times h(E) \rightarrow h(E)$ by $H(h(x), h(y))=$ $A(x, y)$. Since $h: E \rightarrow X$ is one-to-one, it follows that $H$ is well defined. Thus

$$
\begin{aligned}
F_{H(h(x), h(y)), H(h(u), h(v))}(\varphi(t)) \geq & \min \left\{F_{h(x), h(u)}(t), F_{h(y), h(v)}(t), F_{h(x), H(h(x), h(y))}(t),\right. \\
& \left.F_{h(u), H(h(u), h(v))}(t), F_{h(y), H(h(y), h(x))}(t), F_{h(v), H(h(v), h(u))}(t)\right\}
\end{aligned}
$$

for $h(x) \leq h(u), h(v) \leq h(y)$.

We introduce the partial order $\preceq$ on $h(E) \times h(E)$ by

$$
\tilde{a} \preceq \tilde{b} \quad \text { if and only if } \quad h(x) \leq h(u), \quad h(v) \leq h(y),
$$

and the probabilistic metric $\tilde{F}$, for $\tilde{a}=(h(x), h(y)), \tilde{b}=(h(u), h(v)) \in h(E) \times h(E)$, by

$$
\tilde{F}_{\tilde{a}, \tilde{b}}(t):=\min \left\{F_{h(x), g(u)}(t), F_{h(y), h(v)}(t)\right\} .
$$


Since $A$ has the mixed $h$-monotone property, the operator $H$ has the mixed monotone property. Following the results in Corollary 2.1, we conclude that $H$ has a coupled fixed point in $h(E) \times h(E)$, that is,

$$
h(p)=H(h(p), h(q)), \quad h(q)=H(h(q), h(p)) .
$$

Thus there exist $p, q \in X$ such that

$$
h(p)=A(p, q) \quad \text { and } \quad h(q)=A(q, p) .
$$

The proof is complete.

Remark 2.1 Corollary 2.2 improves and generalizes Theorem 7 in [4].

Following similar arguments as in the proof of Theorems 2.1 and 2.2, we can deduce the next result. We omit the details of the proof.

Theorem 2.3 Let $(X, F, \triangle)$ be a complete Menger PM space with a T-norm $\triangle$ of H-type. Suppose $G: X \rightarrow X$ is a mapping satisfying the following: there is a $\varphi \in \Phi$ such that, for all $t>0$ and $x, y \in X$,

$$
F_{G(x), G(y)}(\varphi(t)) \geq \min \left\{F_{x, y}(t), F_{x, G(x)}(t), F_{y, G(y)}(t)\right\} .
$$

Then the operator $G$ has a unique fixed point in X.

Corollary 2.3 ([3], Theorem 1) Let $(X, F, \triangle)$ be a complete Menger PM space with a $T$ norm $\triangle$ of H-type. Suppose $G: X \rightarrow X$ is a mapping satisfying that there is a $\varphi \in \Phi$ such that, for all $t>0$ and $x, y \in X$,

$$
F_{G(x), G(y)}(\varphi(t)) \geq F_{x, y}(t) .
$$

Then the operator $G$ has a unique fixed point $\bar{x}$ in $X$, and, for any $x_{0} \in X, \lim _{n \rightarrow \infty} G^{n}\left(x_{0}\right)=\bar{x}$.

Proof Since

$$
F_{G(x), G(y)}(\varphi(t)) \geq F_{x, y}(t) \geq \min \left\{F_{x, y}(t), F_{x, G(x)}(t), F_{y, G(y)}(t)\right\}
$$

we conclude from Theorem 2.3 that the operator $G$ has a unique fixed point $\bar{x}$ in $X$, and, for any $x_{0} \in X, \lim _{n \rightarrow \infty} G^{n}\left(x_{0}\right)=\bar{x}$.

Corollary 2.4 ([2], Theorem 13) Let $(X, F, \triangle)$ be a complete Menger PM space with a $T$ norm $\triangle$ of H-type. Suppose $G: X \rightarrow X$ is a mapping satisfying, for all $t>0$ and $x, y \in X$,

$$
F_{G(x), G(y)}(\alpha(t) t) \geq F_{x, y}(t)
$$

where $\alpha:(0, \infty) \rightarrow[0,1)$ is a monotonically decreasing function. Then the operator $G$ has a unique fixed point in $X$. 
Proof Set $\varphi(t)=\alpha(t) t$. It is sufficient to prove that $\varphi \in \Phi$. In fact, for all $t>0$, since $\alpha(t)<1$, then $\varphi(t)<t$. On the other hand, for all $n \in Z^{+}$, we see that $0 \leq \varphi^{n+1}(r)=\varphi\left(\varphi^{n}(r)\right)<\varphi^{n}(r)$, thus the sequence $\left\{\varphi^{n}(r)\right\}$ is convergent. Let $\lim _{n \rightarrow+\infty} \varphi^{n}(r)=a \geq 0$, then $a=\lim _{r \rightarrow a+} \varphi(r)$. Suppose $a>0$, then, by the monotony of $\alpha$, we see that

$$
a=\lim _{r \rightarrow a+} \varphi(r)=\lim _{r \rightarrow a+} \alpha(r) r \leq \lim _{r \rightarrow a+} \alpha\left(\frac{a}{2}\right) r=\alpha\left(\frac{a}{2}\right) a<a .
$$

This is a contradiction. Thus $\lim _{n \rightarrow+\infty} \varphi^{n}(r)=0$. This implies that $\varphi \in \Phi$. Then the Corollary 2.4 is a consequence of Theorem 2.3 or Corollary 2.3.

\section{Conclusions}

In this paper, we establish some fixed-point theorems for monotony operators and extend the probabilistic version of the Banach fixed-point principle for general nonlinear contractions to the partially ordered Menger probabilistic metric spaces. Also, we show a sufficient and necessary condition to the existence of the fixed point for a class of monotone operators. As a consequence, our main results improved and extended some recent coupled fixed-point theorems and coincidence-point theorems for the mixed monotone mappings in the literature [2-4].

Finally, we mention two possible applications of our results. One is to the theory of fuzzy metric spaces. Since the difference between Menger PM space and fuzzy metric spaces lies in the different metric, some coincidence-point theorems and fixed-point theorems in fuzzy metric spaces can be obtained under some suitable restrictions. Another possible application of our results is to the theory of random operator equations.

\section{Competing interests}

The author declares that they have no competing interests.

\section{Acknowledgements}

The authors are grateful to the reviewers for their valuable comments and suggestions. This work was partly supported by National Natural Science Foundation of China (11301039).

Received: 9 October 2013 Accepted: 24 January 2014 Published: 24 Feb 2014

\section{References}

1. Sehgal, VM, Bharucha-Reid, AT: Fixed points of contraction mappings on PM-spaces. Math. Syst. Theory 6, 97-102 (1972)

2. Ćirić, L: Solving the Banach fixed point principle for nonlinear contractions in probabilistic metric spaces. Nonlinear Anal. 72, 2009-2018 (2010)

3. Jachymski, J: On probabilistic $\varphi$-contractions on Menger spaces. Nonlinear Anal. 73, 2199-2203 (2010)

4. Ćirić, L, Agarwal, RP, Samet, B: Mixed monotone-generalized contractions in partially ordered probabilistic metric spaces. Fixed Point Theory Appl. 2011, 56 (2011)

5. Bhaskar, TG, Lakshmikantham, V: Fixed point theory in partially ordered metric spaces and applications. Nonlinear Anal. 65, 1379-1393 (2006)

6. Cherichi, M, Samet, B: Fixed point theorems on ordered gauge spaces with applications to nonlinear integral equations. Fixed Point Theory Appl. 2012, 13 (2012)

7. Haghi, RH, Rezapour, Sh, Shahzad, N: Some fixed point generalizations are not real generalizations. Nonlinear Anal. 74, 1799-1803 (2011)

8. Hadzić, O, Pap, E: Fixed Point Theory in Probabilistic Metric Spaces. Kluwer Academic, Dordrecht (2001)

9. Hadzić, O: A fixed point theorem in Menger spaces. Publ. Inst. Math. (Belgr.) 20, 107-112 (1979)

10. Hadzić, O, Ovcin, Z: Fixed point theorems in fuzzy metric and probabilistic metric spaces. Zb. Rad. Prir--Mat. Fak., Ser. Mat. 24, 197-209 (1994)

11. Lakshmikantham, V, Ciric, L: Coupled fixed point theorems for nonlinear contractions in partially ordered metric spaces. Nonlinear Anal. 70, 4341-4349 (2009)

12. Liu, Y, Li, Z: Coincidence point theorems in probabilistic and fuzzy metric spaces. Fuzzy Sets Syst. 158, 58-70 (2007)

13. Liu, $Y$, Wang, $X$ : Contraction conditions with perturbed linear operators and applications. Math. Commun. 15, 25-35 (2010)

14. Liu, Y, Li, Z: Krasnoselskii type fixed point theorems and applications. Proc. Am. Math. Soc. 136, 1213-1220 (2008) 
15. Liu, Y, Li, Z: Schaefer type theorem and periodic solutions of evolution equations. J. Math. Anal. Appl. 316, 237-255 (2006)

16. Mihet, D: Altering distances in probabilistic Menger spaces. Nonlinear Anal. 71, 2734-2738 (2009)

17. Mihet, D: Fixed point theorems in probabilistic metric spaces. Chaos Solitons Fractals 41(2), 1014-1019 (2009)

18. Mihet, D: A note on a paper of Hicks and Rhoades. Nonlinear Anal. 65, 1411-1413 (2006)

19. Abbas, M, Nazir, T, Radenović, S: Common fixed point of generalized weakly contractive maps in partially ordered G-metric spaces. Appl. Math. Comput. 218, 9383-9395 (2012)

20. Rus, IA: Generalized Contractions and Applications. Cluj University Press, Cluj (2001)

21. Saadati, R, Sedghi, S, Shobe, N: Modified intuitionistic fuzzy metric spaces and some fixed point theorems. Chaos Solitons Fractals 38, 36-47 (2006)

22. Samet, B, Rajovic, M, et al.: Common fixed-point results for nonlinear contractions in ordered partial metric spaces. Fixed Point Theory Appl. 2011, 71 (2011)

23. Schweizer, B, Sklar, A: Probabilistic Metric Spaces. North-Holland, New York (1983)

24. O'Regan, D, Saadati, R: Nonlinear contraction theorems in probabilistic spaces. Appl. Math. Comput. 195, 86-93 (2008)

25. Xiao, JZ, Zhu, XH, Cao, YF: Common coupled fixed point results for probabilistic $\phi$-contractions in Menger spaces. Nonlinear Anal. 74, 4589-4600 (2011)

26. Shatanawi, W: Some fixed point results for a generalized $\psi$-weak contraction mappings in orbitally metric spaces. Chaos Solitons Fractals 45, 520-526 (2012)

27. Ume, JS: Fixed point theorems for nonlinear contractions in Menger spaces. Abstr. Appl. Anal. 2011, Article ID 143959 (2011)

$10.1186 / 1687-1812-2014-49$

Cite this article as: Wu: Some fixed-point theorems for mixed monotone operators in partially ordered probabilistic metric spaces. Fixed Point Theory and Applications 2014, 2014:49

\section{Submit your manuscript to a SpringerOpen ${ }^{\ominus}$ journal and benefit from:}

- Convenient online submission

- Rigorous peer review

- Immediate publication on acceptance

- Open access: articles freely available online

- High visibility within the field

- Retaining the copyright to your article 